\title{
A dynamic ion-atom hybrid trap for high-resolution cold-collision studies
}

\author{
P. Eberle* \\ A. D. Dörfler* \\ C. von Planta* \\ K. Ravi* \\ S. Willitsch ${ }^{\dagger *}$
}

August 19, 2016

\begin{abstract}
We present a dynamic ion-atom hybrid trap for studies of cold ion-neutral collisions and reactions with a significantly improved energy resolution compared to previous experiments. Our approach is based on pushing a cloud of laser-cooled Rb atoms through a stationary Coulomb crystal of cold ions using precisely controlled, tunable radiation-pressure forces. We demonstrate the tuning of the atom kinetic energies over an interval ranging from $30 \mathrm{mK}$ up to $350 \mathrm{mK}$ with energy spreads as low as $24 \mathrm{mK}$ inferred from the comparison of experimental time-of-flight measurements with Monte Carlo trajectory simulations. We also demonstrate first applications of our method to the investigation of chemical reactions. Our development opens up perspectives for accurate studies of the energy dependence of the reaction rates, the dynamics and the reaction-product ratios of ion-neutral processes in the cold regime. It also paves the way for the realisation of fully energy- and state-controlled cold-collision experiments.
\end{abstract}

\section{Introduction}

Recent technological advances in the cooling of atoms and molecules have enabled the study of collisions and chemical reactions in the "cold regime" at temperatures below $1 \mathrm{~K}$ [1-5]. For ion-neutral collisions, the lowest energies are currently achieved in hybrid experiments in which traps for cold ions and cold atoms are combined [6-8]. In these experiments, ions are typically stored and cooled in a radiofrequency (RF) trap [2,9] which is overlapped with laser-cooled atoms in a magneto-optical trap (MOT) $[10,11]$. In some experiments, the MOT is replaced by a magnetic or optical dipole trap, in which the atoms can further be cooled and even Bose-Einstein condensed [6,7].

Hybrid trapping technology has enabled the study of ion-neutral collisions and reactions in the cold regime. Over the past years, these experiments helped to unravel the rich and complex chemistry underlying ion-atom collisions at low temperatures and paved the way for the exploration of a range of interesting phenomena. Charge exchange processes have been observed in many systems [12-17]. Reaction rates were found to increase considerably with the electronic excitation of the collision partners [12, 18-20]. It was also established that molecular ions can be formed by radiative association under the cold and dilute conditions of a hybrid trap [12-14,21]. At high atom densities, three-body processes were found to become important $[22,23]$.

One important parameter in reactions studies is the relative energy between the collision partners. The reaction rates, the dynamics and the relative branching ratios of product channels can all depend on the collision energy $[24,25]$. It is therefore highly desirable to study these observables as a function of the collision energy while maintaining a high energy resolution, i.e., a narrow energy spread. In hybrid traps, both the ions and atoms are translationally cold and therefore inherently have low kinetic-energy spreads. However, in a double-trap experiment there is no straightforward way of tuning the kinetic energies without increasing their spread and thereby compromising the energy resolution. For instance, in previous studies the collision energies were varied by increasing the micromotion energy of the ions, i.e., the energy associated with their motion in the oscillating electric field of the RF trap $[13,17,18,26]$. However, because of the oscillatory nature of the micromotion, this approach also vastly increased the collision-energy spread thus obscuring subtle details of the underlying collisional dynamics $[13,21,27]$.

\footnotetext{
*Department of Chemistry, University of Basel. Klingelbergstrasse 80, 4056 Basel, Switzerland

${ }^{\dagger}$ Corresponding author. E-mail stefan.willitsch@unibas.ch
} 
In the present article, we present a new "dynamic" ion-atom hybrid trap which enables the study of ion-atom collisions with a greatly enhanced energy resolution in the cold regime. Our approach is based on the generation of a cloud of cold $\mathrm{Rb}$ atoms in a MOT which is accelerated using tunable radiation pressure forces [28-30]. The atom cloud is pushed through a stationary Coulomb crystal of cold ions with which reactive collisions occur. After passing through the ion crystal, the atom cloud is recaptured in a second MOT position. Reversing the push direction, the atoms can be shuttled back and forth between the two MOT positions and through the Coulomb crystal repeatedly. In this way, the duty cycle of the experiment and the measurement statistics are increased. Owing to the narrow initial energy spread of the cold atoms and their controlled acceleration using pulsed, near-resonant laser beams, traveling clouds of atoms with well-defined and narrowly distributed kinetic energies are generated paving the way for collision experiments with a high energy resolution. Some preliminary results of our experiment were already presented in a previous conference proceedings article [31]. Here, we present a detailed characterisation of the performance of our dynamic hybrid trap and demonstrate first applications to reaction studies.

\section{Methods}

\subsection{Hybrid Trap}

Our hybrid trap consists of linear quadrupole RF trap for ions and a vapour-cell MOT for ${ }^{87} \mathrm{Rb}$ atoms as previously described in Refs. [12,13]. A schematic of the experimental setup is depicted in Fig. 1 (a). The ion trap consisted of four segmented cylindrical electrodes arranged in a quadrupolar configuration [2]. $\mathrm{RF}$ potentials with frequencies of $3.4 \mathrm{MHz}$ and amplitudes of up to $400 \mathrm{~V}$ were applied with alternating polarities between adjacent electrodes to dynamically confine the ions in the plane perpendicular to the longitudinal trap axis. Confinement in the longitudinal direction was achieved by the application of static potentials to the outermost segments of the electrodes. $\mathrm{Ca}^{+}$ions were loaded into the trap by non-resonant 355-nm photoionization of Ca atoms emanating from a resistively heated oven. Upon laser cooling, the ions formed Coulomb crystals of spatially localised ions in the trap [2].

The MOT consisted of a pair of water-cooled coils in anti-Helmholtz configuration embedding the ion trap inside the vacuum chamber and a total of six circularly polarised laser beams. Two laser beams along the $y$ and $z$ axes were each retro-reflected in order to provide counter-propagating pairs of MOT beams [11], see Fig. 1 (a). Along the $x$ axis, two independent laser beams were used for cooling and shuttling the atom cloud, see Sec. 2.3 for details. The MOT was loaded from background Rb vapour constantly replenished by an alkali metal dispenser.

Two separate imaging systems were used for monitoring the laser-cooling fluorescence of both the atoms and the ions. The first one consisted of a microscope (magnification $\approx 10 \mathrm{x}$ ) attached to an electronmultiplying charge-coupled device (EMCCD) camera mounted in the $(x, y)$ plane. It served to image the fluorescence of both the atoms and the ions by switching between two narrow-bandpass optical filters. The second imaging system consisted of a 1:1 relay lens system for projecting the fluorescence of the Rb atoms onto a CMOS (complementary metal-oxide semiconductor) camera and a photomultiplier tube (PMT). It was mounted slightly off the $z$ axis above the chamber perpendicularly to the shuttling axis $x$. For the measurement of time-of-flight (TOF) spectra of the moving atom cloud, a mask with two slits of width $100 \mu \mathrm{m}$ was inserted into the image plane inside the relay lens system. TOF spectra were recorded by monitoring the time delay between the fluorescence signals generated by atoms as they passed underneath the two slits.

\subsection{Laser System}

Laser radiation for cooling both the $\mathrm{Ca}^{+}$ions and the $\mathrm{Rb}$ atoms was produced by commercial externalcavity diode lasers (ECDL, Toptica DL 100 and DL 100 pro). ${ }^{40} \mathrm{Ca}^{+}$ions were laser cooled on the $(4 s){ }^{2} S_{1 / 2} \rightarrow(4 p){ }^{2} P_{1 / 2}$ transition at $397 \mathrm{~nm}$. The laser beam was passed through an acousto-optic modulator $(\mathrm{AOM})$ for fast switching and for shifting its frequency slightly to the red of the atomic resonance for Doppler laser cooling. Another laser beam at $866 \mathrm{~nm}$ was used for repumping population out of the metastable $(3 d)^{2} D_{3 / 2}$ state to close the optical cycle. Both lasers were locked to a highprecision wavemeter (HighFinesse WS/U-30).

The laser setup used for cooling and pushing the ${ }^{87} \mathrm{Rb}$ atoms is shown schematically in Fig. 1 (b). Laser radiation around $780 \mathrm{~nm}$ was produced by an ECDL frequency-locked to a saturated-absorptionspectroscopy signal of $\mathrm{Rb}$ in a vapour cell. The output of the diode laser was amplified by a tapered 

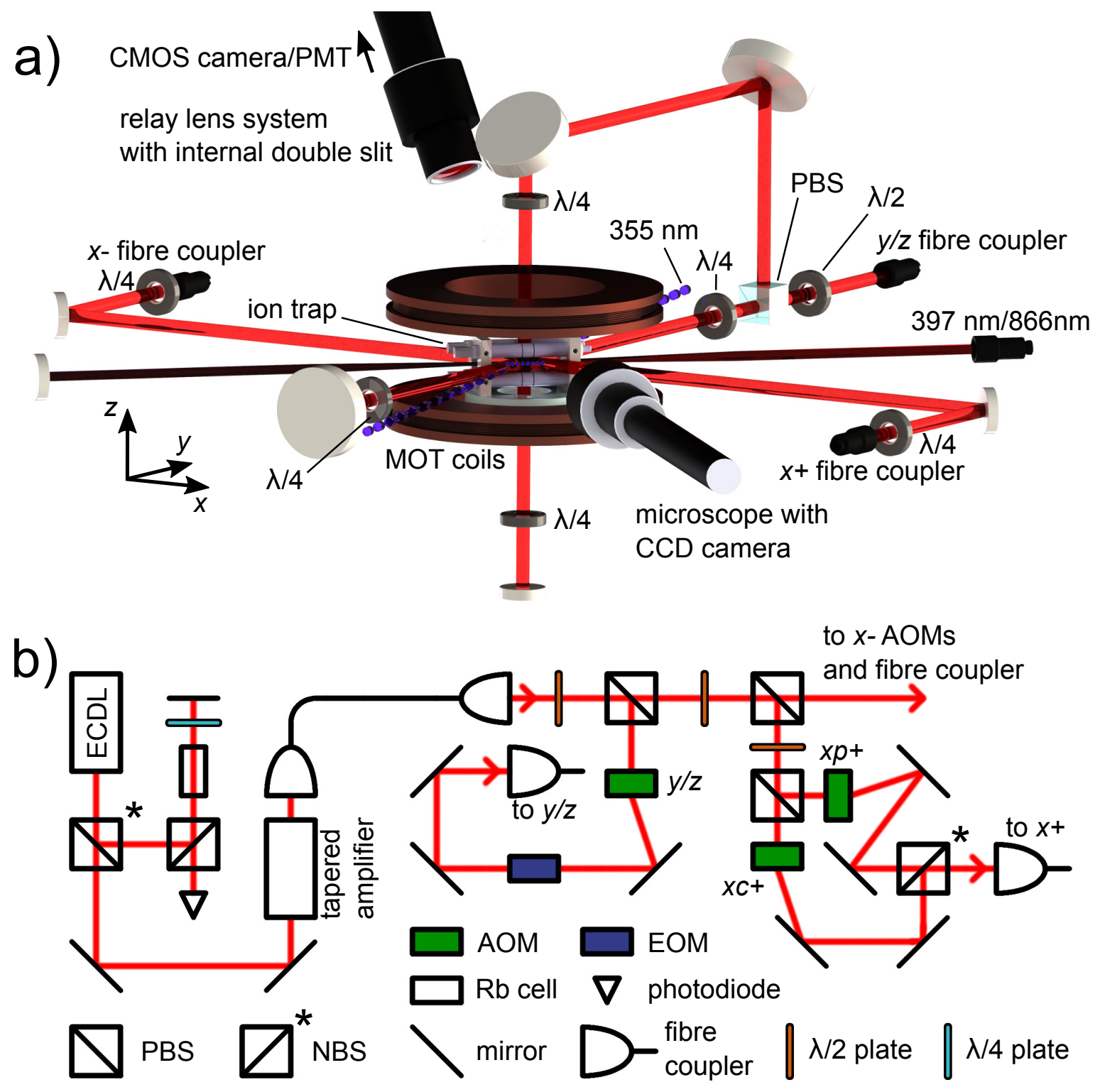

Figure 1: (a) Schematic representation of the hybrid-trap setup. A linear radiofrequency ion trap is embedded in between two coils generating a quadrupolar magnetic field for a magneto-optical trap. Laser beams at $355 \mathrm{~nm}$ as well as $397 \mathrm{~nm}$ and $866 \mathrm{~nm}$ serve to generate and cool ${ }^{40} \mathrm{Ca}^{+}$ions. The red laser beams at a wavelength around $780 \mathrm{~nm}$ are used for the cooling and magneto-optical trapping of ${ }^{87} \mathrm{Rb}$ atoms. Two counter-propagating beams along the $x$ axis can be switched into a configuration for shuttling a cold atom cloud between two trapping positions for studies of collisions between the ions and the atoms. (b) Setup for generating laser radiation around $780 \mathrm{~nm}$ used for cooling and shuttling Rb atoms. See text for details. Abbreviations: ECDL - external-cavity diode laser, AOM - acousto-optic modulator, EOM - electro-optic modulator, PBS - polarising beam splitter, NBS - non-polarising beam splitter.

amplifier (Toptica BoosTA) and then mode-cleaned by coupling into a polarisation-maintaining (PM) optical fibre.

The output of the fibre was split up into two separate beams. The first beam was sent through an $\mathrm{AOM}$ for shifting the frequency slightly to the red of the Rb $(5 s){ }^{2} S_{1 / 2} F=2 \rightarrow(5 p){ }^{2} P_{3 / 2} F=3$ resonance to provide laser radiation for Doppler laser cooling and magneto-optical trapping. The firstorder diffracted output of the AOM was further passed through an electro-optic modulator (EOM) for generating sidebands repumping population on the $\mathrm{Rb}(5 s){ }^{2} S_{1 / 2} F=1 \rightarrow(5 p){ }^{2} P_{3 / 2} F=2$ transition [13]. The laser beam was subsequently coupled into a PM fibre, transported to the experiment 
table and split into two components propagating along the $y$ and $z$ axes of the trap, see Fig. 1 (a).

The second beam was further split into two individual components $x+$ and $x$ - used for cooling and pushing the atoms along both directions of the $x$ axis of the hybrid trap, see Fig. 1 (a). Fig. 1 (b) only shows the branch of the setup for manipulating the $x+$ beam, the one for the $x$ - beam is analogous. The $x+$ beam was further split into two components for cooling $(x c+)$ and pushing $(x p+)$ the atoms. Both beams were passed through separate AOMs to shift their frequencies slightly to the red $(x c+)$ or exactly on-resonance $(x p+)$ of the $(5 s){ }^{2} S_{1 / 2} F=2 \rightarrow(5 p){ }^{2} P_{3 / 2} F=3$ transition and to enable a fast switching of the beams. The first-order-diffracted output of both AOMs was then recombined on a beam splitter and coupled into a PM fibre to be transported to the experiment table. All AOMs were driven by home-made RF generators controlled by a pulse generator (Quantum Composers 9520).

\subsection{Atom-Shuttling Operation}
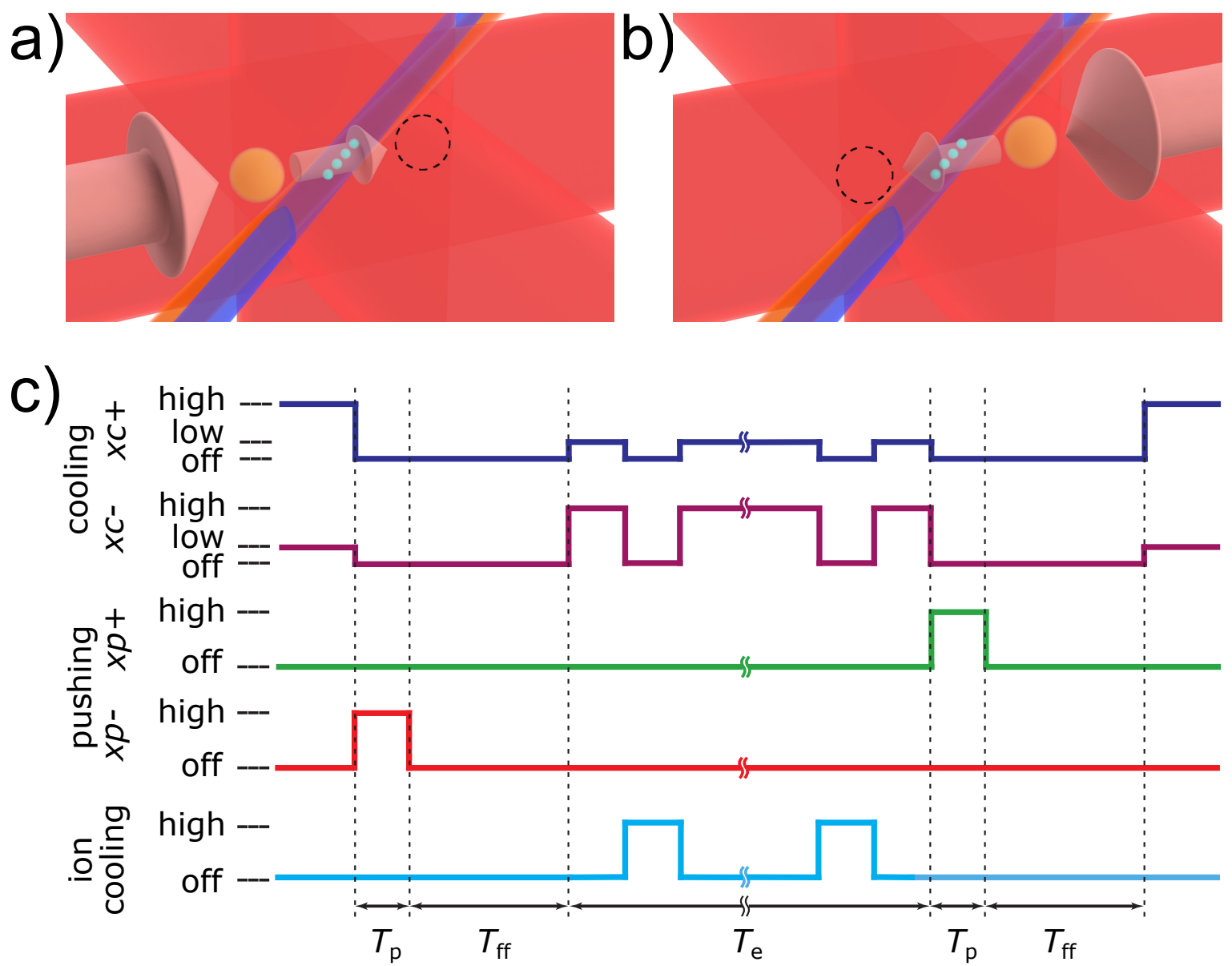

Figure 2: (a) and (b) Schematic representation of the shuttling of a cloud of cold Rb atoms (orange sphere) in between two magneto-optical trapping positions (dashed circles) through a Coulomb crystal of cold ions (blue dots) using radiation pressure forces (large arrows). Laser beams for cooling Rb atoms and $\mathrm{Ca}^{+}$ions are indicated in red and blue, respectively. (c) Timing sequence of laser pulses required for cooling and shuttling the cloud of cold $\mathrm{Rb}$ atoms as well as for cooling the $\mathrm{Ca}^{+}$ions. Abbreviations: $T_{\mathrm{p}}$ - pushing time period, $T_{\mathrm{ff}}$ - free-flight time of the atom cloud, $T_{\mathrm{e}}$ - equilibration period of the atom cloud in MOT. See text for details.

The principle of our dynamic hybrid trap is based on the use of radiation pressure for generating a moving cloud of $\mathrm{Rb}$ atoms with well-defined kinetic energies to collide with stationary cold ions in the RF trap. The experimental procedure for moving the atom cloud is depicted in Figs. 2 (a) and (b), the timing sequence of the laser pulses required is shown in Fig. 2 (c). Initially, a cloud of cold $(T \approx 1 \mathrm{mK}) \mathrm{Rb}$ atoms is produced in a MOT located in an off-centre position (orange sphere in Fig. 1 (a)) by lowering the intensity of one of the cooling laser beams along the $x$ axis. This configuration 
creates a radiation-pressure difference along the $x$ axis which moves the equilibrium position of the atom cloud towards the beam of lower intensity, away from the centre of the hybrid trap.

To move the atoms, the cooling laser beams along the $x$ axis are switched off. A push laser beam $(x p-$ in this example, depicted as a pink arrow in Fig. $2(\mathrm{a}))$ is switched on for a short time to accelerate the atoms towards the hybrid-trap centre. Intensity, detuning and duration of this pulse are adjusted to ensure that the atoms have reached a well-defined constant velocity before they reach the ions. After passing through the Coulomb crystal in the trap centre (blue dots), the atom cloud is re-captured in an opposite off-centre MOT position (indicated by the dashed circle in Fig. 2 (a)) by switching on the $x$-axis cooling beams with a reversed intensity imbalance. After a hold time of typically $40 \mathrm{~ms}$ during which the atoms re-equilibrate in the MOT, the sequence is repeated in the reverse direction (Fig. 2 (b)).

In the current configuration, the atom cloud can be stably shuttled back and forth with a frequency of 25 transits per second. During shuttling, the atoms are constantly laser cooled along the $y$ and $z$ axes to enable the imaging of their fluorescence and to collimate the cloud in the transverse direction. During a time interval of $1 \mathrm{~ms}$ which comprises the acceleration, free flight and re-cooling of the atoms, the $397 \mathrm{~nm}$ laser beam for cooling the $\mathrm{Ca}^{+}$ions is switched off in order to prevent the photoionisation of the $\mathrm{Rb}$ atoms from their excited $(5 p){ }^{2} P_{3 / 2}$ state while they pass through the ion crystal. Similarly, the $780 \mathrm{~nm} \mathrm{Rb}$ and $397 \mathrm{~nm} \mathrm{Ca}^{+}$cooling beams are alternately switched off during the hold period of the atoms in the MOT (see Fig. 2 (c)) to prevent the ionisation of Rb from background vapour.

\subsection{Monte-Carlo Trajectory Simulations of the Moving Atom Cloud}

To simulate the dynamics of the moving atom cloud, a Monte Carlo trajectory-simulation approach was employed. The computational implementation consisted of two separate parts, one for cooling and equilibrating the atoms in a MOT and one for pushing the atom cloud through the interaction region with the ions by radiation pressure forces.

First, a model ultracold atom cloud was simulated with relevant parameters such as laser-beam intensity profiles, laser-frequency detunings and magnetic field gradients fixed to the values used in the experiments. In an ensemble of typically 20'000 Rb atoms, the classical trajectory of each atom was computed by integrating its equations of motion with a Velocity Verlet algorithm. The force model took into account the scattering forces generated by each cooling beam (see Ref. [32] for details) including local Zeeman shifts and effective laser polarisations in the inhomogeneous magnetic field of the MOT. Since the density of the atom cloud was only on the order $10^{8}-10^{9} \mathrm{~cm}^{-3}$, atom-atom interactions were neglected. An additional stochastic heating force was implemented and adjusted to match the experimentally observed thermal and geometric properties of the atom cloud.

After equilibration, the atoms were accelerated by radiation-pressure forces generated by an onresonance push beam towards the centre of the trap while maintaining laser cooling in the transverse direction. In this part, a $4^{t h}$ order Runge-Kutta algorithm was used for the integration of the equations of motion. To enable a comparison with experimental data, TOF spectra (see Sec. 3.1) were simulated by counting the number of atoms passing through the volume sampled by the slit apertures in the experiment as a function of time. From the simulated trajectories, important parameters such as the mean and width of the velocity distribution of the atoms during their transit through the trap centre were determined.

\section{Results}

\subsection{Measurement of Time-of-Flight Spectra}

The kinetic energy of the atoms while passing through the ion crystal is the crucial parameter for applications in collision studies. We measured the velocity of the atoms by recording TOF spectra using the double-slit aperture inserted into the vertical imaging system. The time-resolved fluorescence of the atom cloud passing underneath the slits was recorded by the PMT yielding two peaks in a typical TOF spectrum, see Fig. 3 (a). From the time separation of the two peaks and the distance between the two slits of $950 \mu \mathrm{m}$, the mean velocity and, therefore, kinetic energy of the atom cloud were calculated.

The red dashed line in Fig. 3 (a) shows the result of a Monte-Carlo trajectory simulation using the exact experimental conditions (temperature and position of the initial atom cloud; intensities, intensity profiles and detunings of the cooling and push laser beams; magnetic field distribution of the MOT) as input. The simulated trace has been normalised to the height of the first peak in the experimental 


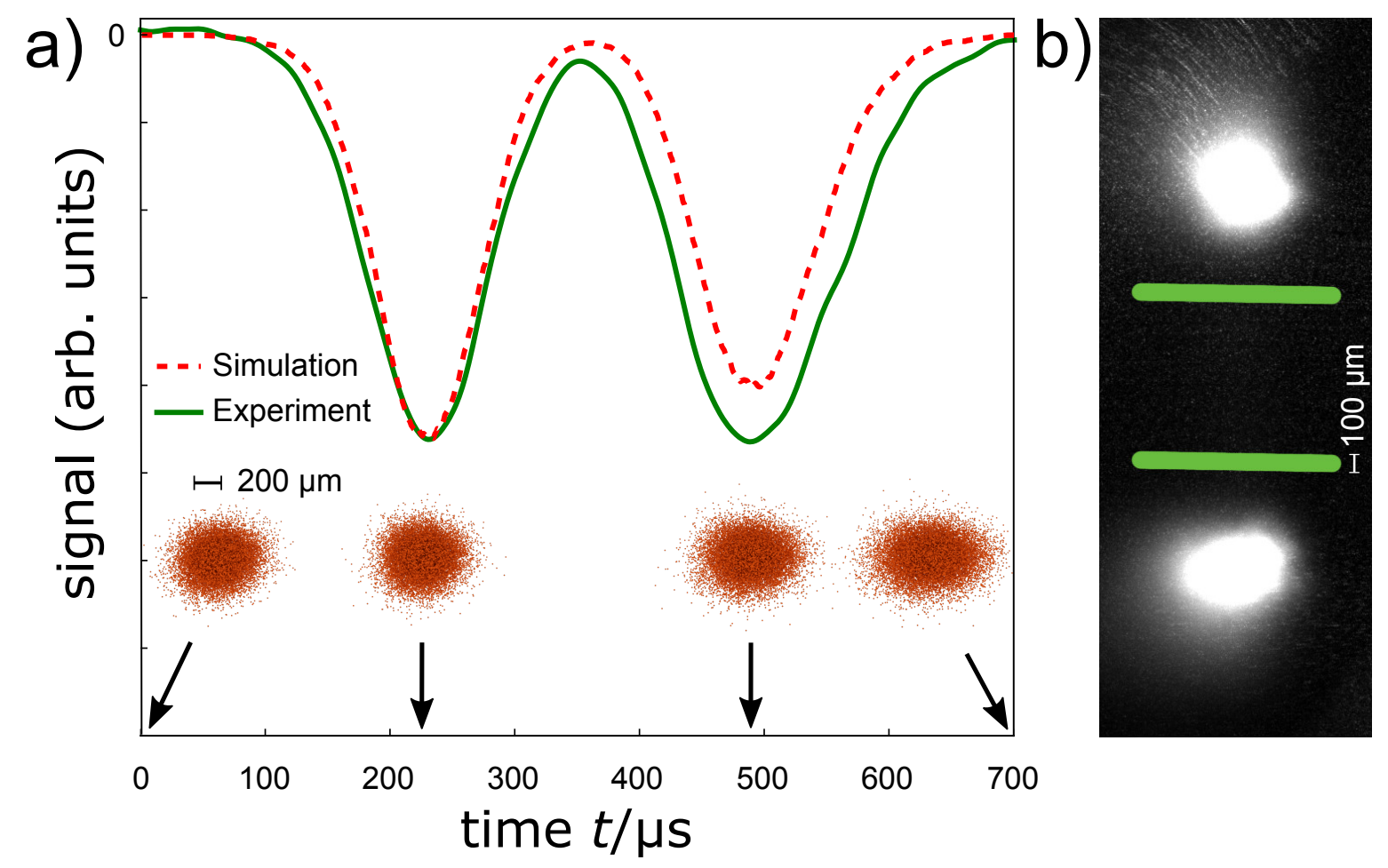

Figure 3: (a) Comparison of an experimental time of flight measurement (solid green line) with the result from Monte-Carlo trajectory simulations (dashed red line). Note that the traces are inverted. In the bottom part of the figure, snapshots from the simulation of the atom cloud during shuttling are shown at specific points in time. See text for details. (b) Image of the shuttling atom cloud recorded with the vertical imaging system. Because the long exposure time of the camera compared to the shuttling period, atoms are imaged in both off-centre MOT positions. The green bars indicate schematically the positions of the slit apertures inserted into the imaging system for time-of-flight measurements.

spectrum. Considering that the simulation did not use any freely adjustable parameters apart from a global scaling factor, the agreement is considered to be good.

In the lower part of the figure, snapshots of the simulated atom positions are shown at specific points in time during shuttling. Both from the TOF traces and simulation snapshots, it can be inferred that the atom cloud slightly expands during the flight until it is recaptured in the opposite MOT position.

Fig. 3 (b) shows a fluorescence image taken with the vertical camera system during the shuttling of atoms. Because the exposure time of the camera $(100 \mathrm{~ms})$ is longer than the shuttling period (40 ms), the image shows atoms in both off-centre MOT positions. The green bars indicate the positions of the slit apertures when they are inserted into the imaging system. Under typical experimental conditions, the distance between the two MOT positions is $\approx 2 \mathrm{~mm}$.

Fig. 4 (a) shows experimental kinetic energies of shuttling Rb atom clouds determined from TOF measurements in both the forward and backward directions (green and blue dots, respectively). The atom kinetic energies were varied by tuning the power of the push laser beams. The atom kinetic energies $E_{\text {kin }}$ have been converted into $\mathrm{Ca}^{+}-\mathrm{Rb}$ collision energies $E_{\text {coll }}$ (bottom abscissa) assuming that the kinetic energies of the $\mathrm{Ca}^{+}$ions can be neglected with respect to the $\mathrm{Rb}$ atoms.

To achieve the same atom kinetic energy in the forward and backward flight direction, slightly different powers for the $x p+$ and $x p$ - beams had to be used, see the right-hand-side ordinate axes in Fig. 4 (a). We attribute this difference to experimental imperfections such as slight misalignments of the laser beams and small variations in the performance of optical components in $x+$ and $x$ - beam paths.

The data shown covers a kinetic-energy interval 40-120 mK. The error bars indicate the standard deviation of a series of 10 measurements. The extensions of the error bars are on the order of the size of the symbols in Fig. 4 (a) illustrating the excellent reproducibility and stability of the experiment which is crucial for achieving a high energy resolution in collision experiments.

The red crosses in Fig. 4 (a) show mean kinetic energies derived from the simulated atom velocity 
a) Rb kinetic energy $E_{\text {kin }} /\left(k_{\mathrm{B}} \mathrm{mK}\right)$
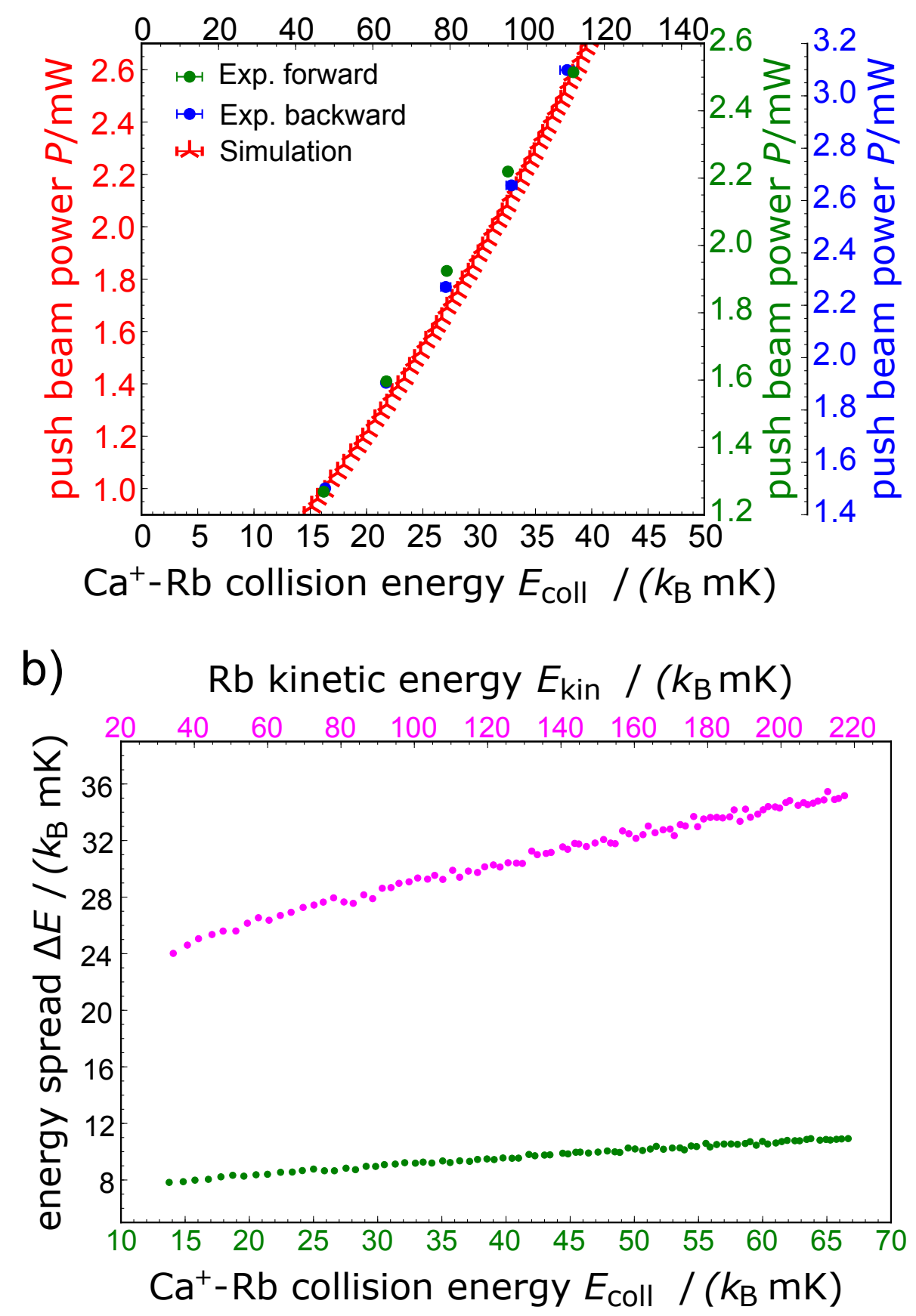

Figure 4: (a) Comparison of experimental Rb kinetic energies $E_{\text {kin }}$ in the forward and backward shuttling direction (green and blue dots, respectively) with simulated kinetic energies (red crosses) as a function of the power of the laser beam used to push the atoms. In the bottom abscissa, the Rb kinetic energies have been converted to $\mathrm{Ca}^{+}-\mathrm{Rb}$ collision energies $E_{\text {coll }}$ assuming that the ions are motionless. See text for details. (b) Energy spreads $\Delta E$ of the Rb atom cloud at the centre of the trap as a function of the kinetic and $\mathrm{Ca}^{+}-\mathrm{Rb}$ collision energy extracted from the full-widths-at-half-maximum of simulated velocity distributions of travelling $\mathrm{Rb}$ atom clouds.

distributions. In these simulations, parameters such as the magnetic field gradients, laser beam diameters, positions and temperature of the initial atom cloud were again fixed to the experimental values and only the push beam power was varied. The simulation results agree well with the observed ones further 
supporting the validity of our computational approach.

A key parameter is the achievable spread of collision energies which impacts the energy resolution of collision experiments. As the widths of the measured time-of-flight profiles of the atoms depend on both, the initial distribution of the atoms and their velocities, the velocity spread of the atom ensemble cannot directly be determined from the TOF profiles. Moreover, the low sensitivity of our camera systems precluded a direct imaging of the cloud size during flight. We thus infer the velocity spread of the atoms from the simulations. Fig. 4 (b) shows the kinetic-energy (magenta dots) and $\mathrm{Ca}^{+}-\mathrm{Rb}$ collisionenergy (green dots) spreads extracted from the full-widths-at-half-maximum (FWHM) of the simulated velocity distributions of the $\mathrm{Rb}$ atoms when they pass the trap centre. The figure covers a range of $\mathrm{Rb}$ kinetic energies from 30-220 $\mathrm{mK}$. The full dynamic range currently achievable in the experiment is $E_{\text {kin }} \approx 30-350 \mathrm{mK}$ in case the Rb cooling lasers are constantly switched on (see Fig. 3 of our preliminary report Ref. [31]). The kinetic-energy spread is simulated to range between $24 \mathrm{mK}$ and $35 \mathrm{mK}$ from the lowest to the highest collision energies in Fig. 4 (b). This corresponds to $\mathrm{Ca}^{+}-\mathrm{Rb}$ collision energy spreads ranging from 8-11 $\mathrm{mK}$ in the collision energy interval from 14-67 $\mathrm{mK}$.

\subsection{Reaction Experiments}

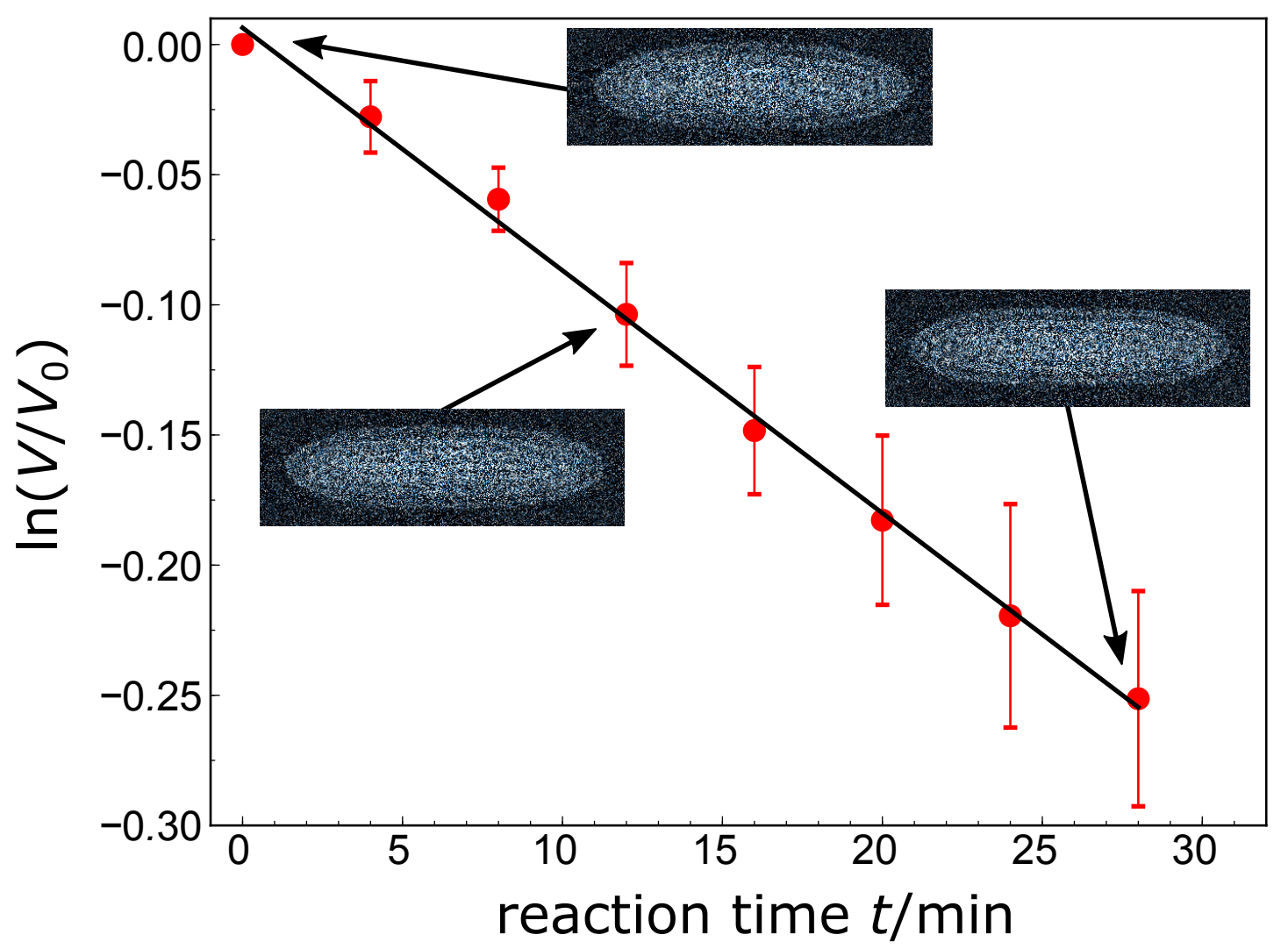

Figure 5: Kinetic data of the reaction of a $\mathrm{Ca}^{+}$Coulomb crystal with a shuttling cloud of $\mathrm{Rb}$ atoms at $\mathrm{Rb}$ kinetic energies of $80 \mathrm{mK}$. The plot shows the logarithm of the relative volume $V / V_{0}$ of the Coulomb crystals as a function of the reaction time. The reaction rate constant is obtained from a fit of an integrated pseudo-first-order rate law to the experimental data, see text for details. The data points represent the average of four experiments and the error bars their $\pm 1 \sigma$ statistical uncertainty. Insets: fluorescence images of $\mathrm{Ca}^{+}$Coulomb crystals taken at various points in time during the reaction experiment.

To demonstrate the applicability of our new setup to studies of chemical reactions, experiments on reactive collisions of the velocity-controlled $\mathrm{Rb}$ atoms with large Coulomb crystals of $\mathrm{Ca}^{+}$ions were performed. Fig. 5 shows a plot of the time-dependent volume of $\mathrm{Ca}^{+}$crystals reacting with shuttling $\mathrm{Rb}$ 
atom clouds. A decrease in the apparent crystal size was observed with reaction time due to fluorescing $\mathrm{Ca}^{+}$ions being replaced by dark product ions [12]. This is also illustrated by the fluorescence images of $\mathrm{Ca}^{+}$crystals shown as insets in Fig. 5. Since atoms were continuously loaded from background vapour, the Rb density was constant and the reaction followed pseudo-first-order kinetics characterized by an exponential decay of the ion number with time [13]. The pseudo-first-order rate constant $k_{p f o}$ was obtained from a linear fit to the logarithm of the relative crystal volume $V / V_{0}$ against time. At a $\mathrm{Rb}$ kinetic energy of $80 \mathrm{mK}$, we determined a pseudo-first-order rate constant $k_{p f o}=1.6(1) \times 10^{-4} \mathrm{~s}^{-1}$ averaged over four experiments. For comparison, the rate constant obtained without the presence of the shuttling $\mathrm{Rb}$ cloud reflecting reactions of $\mathrm{Ca}^{+}$with background gases in the ultrahigh-vacuum chamber was measured to be $5(2) \times 10^{-5} \mathrm{~s}^{-1}$.

\section{Discussion}

Fig. 5 can be compared with Fig. 5 (c) in Ref. [13] showing the analogous experiment in the previous hybrid-trap setup in which the cloud of ultracold $\mathrm{Rb}$ atoms was permanently overlaid with the $\mathrm{Ca}^{+}$ crystal. The effective reaction times in the current experiments are about two order of magnitudes longer than with the stationary MOT owing to the reduced contact time between the atoms and the ions. Indeed, as the atoms repeatedly shuttle through the ion crystal, the effective contact time is only on the order of a few ms at 25 atom transits per second. The present mode of operation in which the atom cloud is repeatedly shuttled back and forth through the ion crystal is therefore imperative to increase the duty cycle of the experiment.

Large Coulomb crystals such as the ones used in the experiments shown in Fig. 5 exhibit broad kinetic-energy distributions of the ions due to the spread of micromotion energies and are thus not suitable for high-resolution collision experiments. Instead, it is preferable to use strings of ions located on the central axis of the ion trap. In this configuration, the micromotion energies are minimised and the ions can be assumed to have negligible kinetic energies compared to the moving atoms [13, 27]. However, due to the necessarily small number of ions in this case, collision events are rare and extensive averaging is required. Thus, the present approach is primarily suited to probe fast reactions for which the measurement times can be kept within practicable limits.

In essence, our shuttling atom cloud represents a highly controlled implementation of an atomic beam. In this sense, our approach relies on the replacement of the previous double-trap experiment with its poorly controllable energy distributions by a combined beam-and-trap experiment featuring a superior control over the particle energies. The present setup can readily be used for the measurement of the variation of pseudo-first-order reaction rates with the collision energy. The determination of absolute bimolecular rate constants, however, requires the accurate calibration of the Rb densities in the interaction region which is notoriously challenging for beam experiments [33]. Procedures for an accurate determination of the $\mathrm{Rb}$ densities in the beam are currently being developed and will be reported elsewhere.

In the current setup, the average kinetic energy of the moving Rb atom cloud can be tuned from 30-350 $\mathrm{mK}$ with a spread as low as $24 \mathrm{mK}$. This implies a significant improvement in terms of tunability and control of the atom energies for cold-ion-chemistry experiments which opens the door for precise studies of the energy dependence of ion-neutral reactive processes in the cold regime. Our simulations suggest that the presently observed spread of Rb kinetic energies is mainly caused by two effects. First, the finite temperature of the initial atom cloud entails a distribution of velocities of the trapped atoms. Thus, every atom experiences a slightly different Doppler shift and therefore excitation rate of the push laser beam, leading to different accelerations. Reducing the temperature of the initial atom cloud should mitigate this problem. Second, the finite size of the initial atom cloud across the Gaussian intensity profile of the push laser beam entails reduced acceleration rates of atoms at the extremities of the cloud. As atoms in these positions are also the hottest in the trap, this reinforces the repercussions of the finite cloud temperature and leads to a further increase of the velocity spread of the ensemble after acceleration. The use of shaped laser beams with a flatter intensity profile for pushing should reduce this effect.

Finally, as an alternative to the present implementation of a dynamic hybrid trap one could consider reversing the role of atoms and ions and shuttle the ions through a stationary atom cloud. This approach would require controlling the kinetic energies of the ions on the $\mu \mathrm{eV}$ level during shuttling to reach a performance comparable with the present approach. Such an exquisite degree of control has recently been achieved using advanced ion trap designs and trap electronics optimised for precise ion shuttling [34]. 


\section{$5 \quad$ Summary and Outlook}

We have developed and characterised a dynamic ion-atom hybrid trap which paves the way for coldcollision and reaction studies with a significantly enhanced control over the particle energies compared to previous implementations. Our approach relies on pushing a cloud of laser-cooled Rb atoms through a Coulomb crystal of cold ions using well-defined, tunable radiation-pressure forces. Good agreement was found between time-of-flight measurements of moving Rb clouds with the results of Monte-Carlo trajectory simulations. It was shown that the atom kinetic energies can be tuned over an interval ranging approximately from $30 \mathrm{mK}$ to $350 \mathrm{mK}$ with a simulated spread as low as $24 \mathrm{mK}$.

The current development opens up perspectives to probe ion-neutral collisions with tunable energies in the cold regime. Prospective applications include studies of the energy dependence of ion-neutral processes revealing subtle details of intermolecular interactions [18] and possibly collisional quantum features such as scattering resonances [21] both of which have recently been observed in cold collisions between neutral species $[4,35,36]$. As already demonstrated in Ref. [18], the present experiments are not limited to atomic ions, but can readily be extended to sympathetically cooled molecular species. In combination with techniques for the state preparation of the molecular ions, see, e.g., Ref. [37], energy and state controlled cold-chemistry experiments are now within reach paving the way for the characterisation of cold ion-neutral processes at an unprecedented level of detail.

\section{Acknowledgements}

This work is supported by the Swiss National Science Foundation, grant nr. 200021_156182, and the European Commission under the Seventh Framework Programme FP7 GA 607491 COMIQ.

\section{References}

[1] G. Quéméner and P. S. Julienne. Chem. Rev., 112:4949, 2012.

[2] S. Willitsch. Int. Rev. Phys. Chem., 31:175, 2012.

[3] B. K. Stuhl, M. T. Hummon, and J. Ye. Annu. Rev. Phys. Chem., 65:501, 2014.

[4] J. Jankunas and A. Osterwalder. Annu. Rev. Phys. Chem., 66:241, 2015.

[5] B. Heazlewood and T. P. Softley. Annu. Rev. Phys. Chem., 66:475, 2015.

[6] A. Härter and J. Hecker Denschlag. Contemp. Phys., 55:33, 2014.

[7] C. Sias and M. Köhl. Quantum Gas Experiments, page 267. World Scientific Publishing, Singapore, 2014.

[8] S. Willitsch. Proc. Int. Sch. Phys. Enrico Fermi, 189:255, 2015.

[9] F. G. Major, V. N. Gheorghe, and G. Werth. Charged Particle Traps. Springer, Berlin and Heidelberg, 2005.

[10] E. L. Raab, M. Prentiss, A. Cable, S. Chu, and D. E. Pritchard. Phys. Rev. Lett., 59:2631, 1987.

[11] C. F. Foot. Atomic Physics. Oxford University Press, Oxford, 2005.

[12] F. H. J. Hall, M. Aymar, N. Bouloufa-Maafa, O. Dulieu, and S. Willitsch. Phys. Rev. Lett., 107:243202, 2011.

[13] F. H. J. Hall, P. Eberle, G. Hegi, M. Raoult, M. Aymar, O. Dulieu, and S. Willitsch. Mol. Phys., 111:2020, 2013.

[14] F. H. J. Hall, M. Aymar, M. Raoult, O. Dulieu, and S. Willitsch. Mol. Phys., 111:1683, 2013.

[15] C. Zipkes, S. Palzer, L. Ratschbacher, C. Sias, and M. Köhl. Phys. Rev. Lett., 105:133201, 2010.

[16] W. G. Rellergert, S. T. Sullivan, S. Kotochigova, A. Petrov, K. Chen, S. J. Schowalter, and E. R. Hudson. Phys. Rev. Lett., 107:243201, 2011. 
[17] S. Schmid, A. Härter, and J. Hecker Denschlag. Phys. Rev. Lett., 105:133202, 2010.

[18] F. H. J. Hall and S. Willitsch. Phys. Rev. Lett., 109:233202, 2012.

[19] L. Ratschbacher, C. Zipkes, C. Sias, and M. Köhl. Nat. Phys., 8:649, 2012.

[20] S. T. Sullivan, W. G. Rellergert, S. Kotochigova, and E. R. Hudson. Phys. Rev. Lett., 109:223002, 2012.

[21] H. da Silva jr., M. Raoult, M. Aymar, and O. Dulieu. New J. Phys., 17:045015, 2015.

[22] A. Härter, A. Krükow, A. Brunner, W. Schnitzler, S. Schmid, and J. Hecker Denschlag. Phys. Rev. Lett., 109:123201, 2012.

[23] A. Härter, A. Krükow, M. Deiß, B. Drews, E. Tiemann, and J. Hecker Denschlag. Nat. Phys., 9:512, 2013.

[24] R. D. Levine. Molecular Reaction Dynamics. Cambridge University Press, Cambridge, 2005.

[25] M. Brouard and C. Vallance, editors. Tutorials in Molecular Reaction Dynamics. The Royal Society of Chemistry, Oxford, 2012.

[26] C. Zipkes, S. Palzer, C. Sias, and M. Köhl. Nature, 464:388, 2010.

[27] M. T. Bell, A. D. Gingell, J. Oldham, T. P. Softley, and S. Willitsch. Faraday Discuss., 142:73, 2009.

[28] Z. T. Lu, K. L. Corwin, M. J. Renn, M. H. Anderson, E. A. Cornell, and C. E. Wieman. Phys. Rev. Lett., 77:3331, 1996.

[29] W. Wohlleben, F. Chevy, K. Madison, and J. Dalibard. Eur. Phys. J. D, 15:237, 2001.

[30] E. Dimova, O. Morizot, G. Stern, C. L. Garrido Alzar, A. Fioretti, V. Lorent, D. Comaprat, H. Perrin, and P. Pillet. Eur. Phys. J. D, 42:299, 2007.

[31] P. Eberle, A.D. Dörfler, C. von Planta, K. Ravi, D. Haas, D. Zhang, S.Y. T. van de Meerakker, and S. Willitsch. J. Phys. Conf. Ser., 635:012012, 2015.

[32] I. Rouse and S. Willitsch. Phys. Rev. A, 92:053420, 2015.

[33] D. Rösch, S. Willitsch, Y.-P. Chang, and J. Küpper. J. Chem. Phys., 140:124202, 2014.

[34] L. E. de Clercq, H.-Y. Lo, M. Marinelli, D. Nadlinger, R. Oswald, V. Negnevitsky, D. Kienzler, B. Keitch, and J. P. Home. Phys. Rev. Lett., 116:080502, 2016.

[35] A. B. Henson, S. Gersten, Y. Shagam, J. Narevicius, and E. Narevicius. Science, 338:234, 2012.

[36] Y. Shagam, A. Klein, W. Skomorowski, R. Yun, V. Averbukh, C. P. Koch, and E. Narevicius. Nat. Chem., 7:921, 2015.

[37] X. Tong, A. H. Winney, and S. Willitsch. Phys. Rev. Lett., 105:143001, 2010. 